\title{
Electrostatic double-layer interaction between stacked charged bilayers
}

\author{
Mafumi Hishida, ${ }^{1, *}$ Yoko Nomura, ${ }^{1}$ Ryo Akiyama, ${ }^{2}$ Yasuhisa Yamamura, ${ }^{1}$ and Kazuya Saito ${ }^{1, \dagger}$ \\ ${ }^{1}$ Department of Chemistry, Faculty of Pure and Applied Sciences, University of Tsukuba, Tsukuba, Ibaraki 305-8571, Japan \\ ${ }^{2}$ Department of Chemistry, Kyushu University, Fukuoka 819-0395, Japan
}

(Received 31 August 2017; published 19 October 2017)

\begin{abstract}
The inapplicability of the DLVO theory to multilayered anionic bilayers is found in terms of the co-ion-valence dependence of the lamellar repeat distance. Most of the added salt is expelled from the interlamellar space to the bulk due to the Gibbs-Donnan effect on multiple bilayers with the bulk. The electrostatic double-layer interaction is well expressed by the formula recently proposed by Trefalt. The osmotic pressure due to the expelled ions, rather than the van der Waals interaction, is the main origin of the attractive force between the bilayers.
\end{abstract}

DOI: 10.1103/PhysRevE.96.040601

Dispersion and aggregation of charged colloids have long been interpreted using the Derjaguin-Landau-VerweyOverbeek (DLVO) theory, i.e., the electrostatic double-layer (DL) interaction and the van der Waals (vdW) interaction are balanced and the distance between the colloids is obtained from the position of the potential minimum [1-3]. The DL interaction originates from an entropic force due to the heterogeneous ion distribution. Based on the pioneering studies by Gouy, Chapman, Debye, and Hückel, the DL interaction has been formulated by solving the Poisson-Boltzmann equation with some approximations [4-8]. It is emphasized that the Poisson-Boltzmann treatment is a continuum approach, in which the solvent is regarded as a mere homogeneous background.

In an electrolyte solution of relatively high concentration, the DL interaction is formulated for surfaces with low surface potential (Debye-Hückel approximation) as $[8,9]$

$$
\begin{aligned}
U_{\mathrm{DL}}(x) & =\frac{\sigma^{2} \lambda_{D}}{\varepsilon_{0} \varepsilon}\left[\operatorname{coth}\left(x / 2 \lambda_{D}\right)-1\right] \\
& \approx \frac{2 \sigma^{2} \lambda_{D}}{\varepsilon_{0} \varepsilon} e^{-x / \lambda_{D}}
\end{aligned}
$$

while for surfaces with rather large surface potential but weak coupling between two surfaces, it is formulated as $[8,9]$

$$
U_{\mathrm{DL}}(x)=\frac{32 \varepsilon_{0} \varepsilon}{e_{0}^{2} \beta^{2} \lambda_{D}} e^{-x / \lambda_{D}} .
$$

where $\varepsilon_{0} \varepsilon$ is the dielectric constant of an aqueous phase, $\beta=1 / k T$ is the inverse thermal energy, $e_{0}$ is the elementary charge, and $\sigma$ is the surface charge density. The Debye screening length $\lambda_{\mathrm{D}}$ in the electrolyte solution with ionic strength $I$ is given as

$$
\lambda_{\mathrm{D}}=\sqrt{\frac{\varepsilon_{0} \varepsilon}{2 \beta e_{0}^{2} I}} .
$$

These formulas of DL interaction indicate that the DL interaction is determined only by $I$, irrespective of the nature or charges of the ions.

The above formulas of the DL interaction have been obtained analytically under conditions where the electrolyte

\footnotetext{
*hishida@chem.tsukuba.ac.jp

${ }^{\dagger}$ kazuya@chem.tsukuba.ac.jp
}

solution is composed of $Z: Z$ ions [3,9] (the valences of counterion and co-ion are the same). The formulas have been validated by surface force measurements [10-12]. However, cases with asymmetric ion valences have not been fully discussed, except for $2 Z: Z$ ions [9], although most colloidal systems, including biomolecular ones, correspond to the asymmetric cases.

On the other hand, Trefalt et al. [13-19] have recently investigated the critical coagulation concentration of charged colloids in electrolyte solutions with $1: Z$ ions (monovalent counterion and multivalent co-ion) and have reported that aggregation behavior depends on the co-ion valence, even though the ionic strengths are the same. Though a similar behavior, i.e., more effective aggregation with smaller co-ion valence, has been reported for the coil-globule transition of DNA [20,21], there have been only a few reports on colloidal aggregation in electrolyte solutions with $1: Z$ ions. Trefalt [16] suggested that the expulsion of co-ions from the gap between colloids, which has not been considered in the classical derivation of DL interactions in an electrolyte solution [Eqs. (1) and (2)] via Poisson-Boltzmann treatment with some approximations, is the origin of the dependence on the co-ion valences. However, the expulsion of co-ions has not been fully verified, and the mechanism should be further researched.

In the present Rapid Communication, the expulsion is confirmed from the co-ion valence dependence on the lamellar repeat distance $d$ of freely suspended charged bilayers. The $d$ value, precisely measured by x-ray diffraction, enables discussion on the interaction potentials over a wide range of ionic strengths [22-25], in contrast to the studies on critical coagulation concentration of spherical colloids or the coilglobule transition of DNA. The mechanism of the expulsion is explained in terms of the so-called Gibbs-Donnan effect on aggregated colloids, implying different DL interactions between a multilayered system and a system with only a couple of bilayers.

An anionic lipid, 1,2-dioleoyl-sn-glycero-3-phospho-Lserine (DOPS, sodium salt), was purchased from Avanti Polar Lipid, Inc. Three salts having the same counterion but different co-ions, $\mathrm{NaCl}$ ( $>99.5 \%$, from Nacalai Tesque, Inc.), $\mathrm{Na}_{2} \mathrm{SO}_{4}(>99.0 \%$, from Wako Pure Chemical Industries, Ltd.), and $\mathrm{Na}_{3} \mathrm{PO}_{4} \cdot 12 \mathrm{H}_{2} \mathrm{O}(>98.0 \%$, from Wako Pure Chemical Industries, Ltd.) were used. These reagents were used without further purification. Multistep dissociation of $\mathrm{Na}_{3} \mathrm{PO}_{4}$ in water will be discussed later. DOPS was first dissolved in an organic 
solvent (chloroform:methanol $=2: 1 \mathrm{v} / \mathrm{v}$ ) and the solvent was evaporated in vacuum to obtain the DOPS dry film, onto which aqueous solution of each salt was poured and the solutions were vortexed. Resultant DOPS concentrations were about $60 \mathrm{mM}$ (4.7 vol\%), indicating that the solutions inevitably contain $60 \mathrm{mM}$ of counterions $\left(\mathrm{Na}^{+}\right)$even without salt addition. The DOPS:salt solutions were frozen and molten, and then sonicated using a probe-type sonicator (UH-50 from SMT Co., Ltd.). The freeze-melt-sonication treatments were repeated eight times to obtain equilibrium dispersion of ions. During these treatments, lipid bilayers should be largely disturbed and the ions should be free to penetrate the lipid bilayer and reach equilibrium distribution. Without the treatments, however, multiple Bragg peaks were observed by small-angle x-ray scattering (SAXS) due to the inhomogeneous ion distribution.

Ion concentrations are defined as follows: $\rho_{0}=60 \mathrm{mM}$ is the concentration of the counterion $\left(\mathrm{Na}^{+}\right)$originally stuck to DOPS. $\rho$ is the added salt concentration. When the ion valences are [counterion]:[co-ion] $=1: Z$, the counterion and co-ion concentrations are $\rho Z$ and $\rho$, respectively. The total counterion concentration is $\rho_{\mathrm{Na}}=\rho_{0}+\rho Z$. The ionic strength of the solution $I$ is given by $[3,8]$

$$
I=\sum_{i} \frac{1}{2} \rho_{i} Z_{i}^{2}=\frac{1}{2}\left(\rho_{0}+\rho Z+\rho Z^{2}\right),
$$

where $Z_{i}$ is the valence of the ionic species $i$. In the present experiment, $I$ ranges between $30(\rho=0 \mathrm{mM})$ and ca. 2000 $\mathrm{mM}$. Since $\mathrm{PO}_{4}^{3-}$ equilibrates with $\mathrm{HPO}_{4}^{2-}+\mathrm{OH}^{-}$with the equilibrium constant $K_{\mathrm{b}}=2 \times 10^{-2} \mathrm{~mol} / \mathrm{L}$, the final form of $I$ is modified to $I=\frac{1}{2}\left(\rho_{0}+12 \rho+2 K_{\mathrm{b}}-2 \sqrt{4 \rho K_{\mathrm{b}}+K_{\mathrm{b}}^{2}}\right)$ in the case of $\mathrm{Na}_{3} \mathrm{PO}_{4}$.

Small-angle $\mathrm{X}$-ray scattering (SAXS) experiments were performed at BL6A and BL10C, Photon factory, KEK, Japan. The X-ray wavelengths were 1.48-1.7 $\AA$, and the distances between the samples and the detector were 2-3 m. The distances were calibrated with a standard sample (silver behenate). The detectors were PILATUS 300K and PILATUS3 2M (DECTRIS Ltd.) at BL6A and BL10C, respectively. Available $q$ range was $0.005-0.16 \AA^{-1}$. We further performed SAXS at BL10C with shorter-wavelength X-ray $(0.89 \AA)$ to record the scattering pattern in a wide $q$ range $\left(0.02-0.37 \AA^{-1}\right)$, from which the form factors of the lipid bilayers in saline solutions were evaluated [see Fig. 1(a)]. All measurements were performed at $25^{\circ} \mathrm{C}$, at which the DOPS bilayer is in the liquid-crystalline phase.

$\zeta$ potentials (the electrostatic potential at a slipping plane) of the vesicles of DOPS bilayers were measured using a microscope-type $\zeta$ potential meter (ZEECOM ZC-3000, Microtec Co., Ltd.), in the same manner as previously reported [24]. The lipid concentrations were kept at $60 \mu \mathrm{M}$, because it was hard to measure $\zeta$ potential of more concentrated samples. Therefore, $\rho_{\mathrm{Na}} \approx \rho Z$. The voltage for the electrophoresis could not be applied appropriately on the solution with high ion concentration because electric current flowed in the solution. The limit of $\rho_{\mathrm{Na}}$ was $100-150 \mathrm{mM}$.

SAXS intensity $J(q)$ is generally expressed as [26]

$$
J(q)-B_{\mathrm{G}} \propto \frac{|F(q)|^{2} S(q)}{q^{2}},
$$
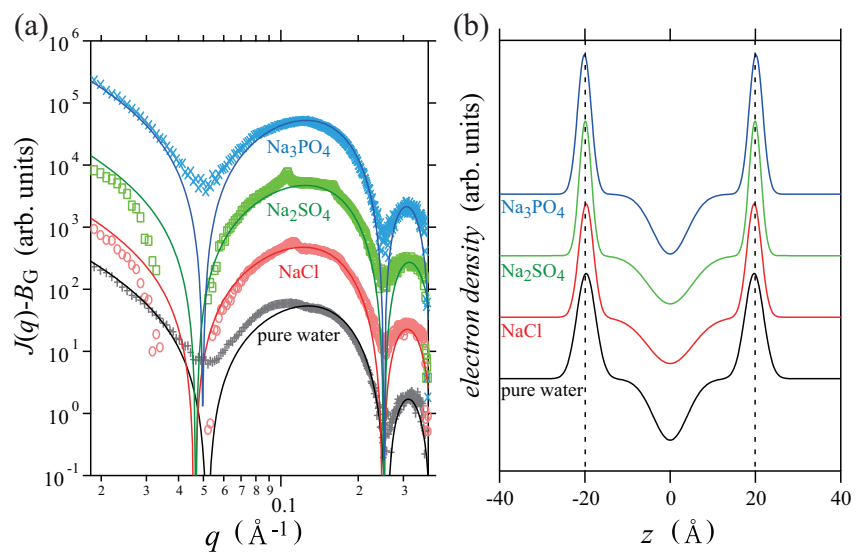

FIG. 1. (a) SAXS intensities without background $\left(J(q)-B_{\mathrm{G}}\right)$, over a wide $q$ range in $\mathrm{NaCl}$ (red), $\mathrm{Na}_{2} \mathrm{SO}_{4}$ (green), and $\mathrm{Na}_{3} \mathrm{PO}_{4}$ (blue) solutions and pure water (black). The added $\mathrm{Na}^{+}$concentration is $\rho Z=30 \mathrm{mM}$. Solid lines are the results of fitting. (b) Deduced electron density profiles of DOPS bilayers in the three saline solutions. Dashed lines are located at $\pm 19.9 \AA$.

where $F(q), S(q)$, and $B_{\mathrm{G}}$ stand for the form factor, the structure factor, and background, respectively. In the present case, $S(q)$ corresponds to the Bragg peaks due to the multilamellar structure. $d$ was mainly obtained from the positions of the first Bragg peaks $(q=2 \pi / d)$. However, the first Bragg peak is annihilated when it locates between $q=0.04-0.06 \AA^{-1}$, where $|F(q)|^{2}$ of the DOPS bilayer is very small (Fig. 1). In these cases, we evaluated $d$ from the position of the second Bragg peak.

The observed $d$ is the sum of the water layer thickness $d_{\mathrm{w}}$ and the bilayer thickness $d_{\mathrm{b}}$ [22,23]. On the other hand, the interactions between the lipid bilayers determine $d_{\mathrm{w}}$. To obtain the dependence of $d_{\mathrm{w}}$ on $I, d_{\mathrm{b}}$ was evaluated from the form factor $F(q)$. Figure 1(a) shows $J(q)-B_{\mathrm{G}}$ for the added counterion concentration $\rho Z=30 \mathrm{mM}(I=60.0$, 75.0, and 75.4 mM for $\mathrm{NaCl}, \mathrm{Na}_{2} \mathrm{SO}_{4}$, and $\mathrm{Na}_{3} \mathrm{PO}_{4}$ solutions, respectively). Since the Bragg peaks are relatively weak in these scattering patterns, we can approximate $S(q) \approx 1$. Then, the $J(q)-B_{\mathrm{G}}$ is fitted using $F(q)$ for a bilayer [26], shown as the solid lines in Fig. 1. From the fitting, the electron density profiles of a DOPS bilayer in three saline solutions $(\rho Z=$ $30 \mathrm{mM}$ ) are estimated [Fig. 1(b)]. The bilayer thickness $d_{\mathrm{b}}$ is found to be almost constant $[(39.8 \pm 0.4) \AA]$ irrespective of the salt additions. It is thus concluded that the bilayer structure hardly changes in these saline solutions.

Figure 2 shows the water layer thickness $d_{\mathrm{w}}=d-d_{\mathrm{b}}$ against $I$ in the three saline solutions with different co-ions. $d_{\mathrm{w}}$ clearly depends on the valence of co-ions. The $d_{\mathrm{w}}$ increases with increasing co-ion valence at the same $I$. This dependence on the co-ion valences agrees with reported trends in the critical coagulation concentration of charged colloids [13-19] and the coil-globule transition of DNA [20,21]. With increasing $I, d_{\mathrm{w}}$ decreases and the $I$ dependence of $d_{\mathrm{w}}$ reaches $d_{\mathrm{w}} \propto I^{-\frac{1}{2}}$.

For bilayers with a thickness $d_{\mathrm{b}}$, the vdW interaction [22] is given by

$$
U_{\mathrm{vdW}}\left(d_{\mathrm{w}}\right)=-\frac{H}{12 \pi}\left[\frac{1}{d_{\mathrm{w}}^{2}}-\frac{2}{\left(d_{\mathrm{w}}+d_{\mathrm{b}}\right)^{2}}+\frac{1}{\left(d_{\mathrm{w}}+2 d_{\mathrm{b}}\right)^{2}}\right] .
$$




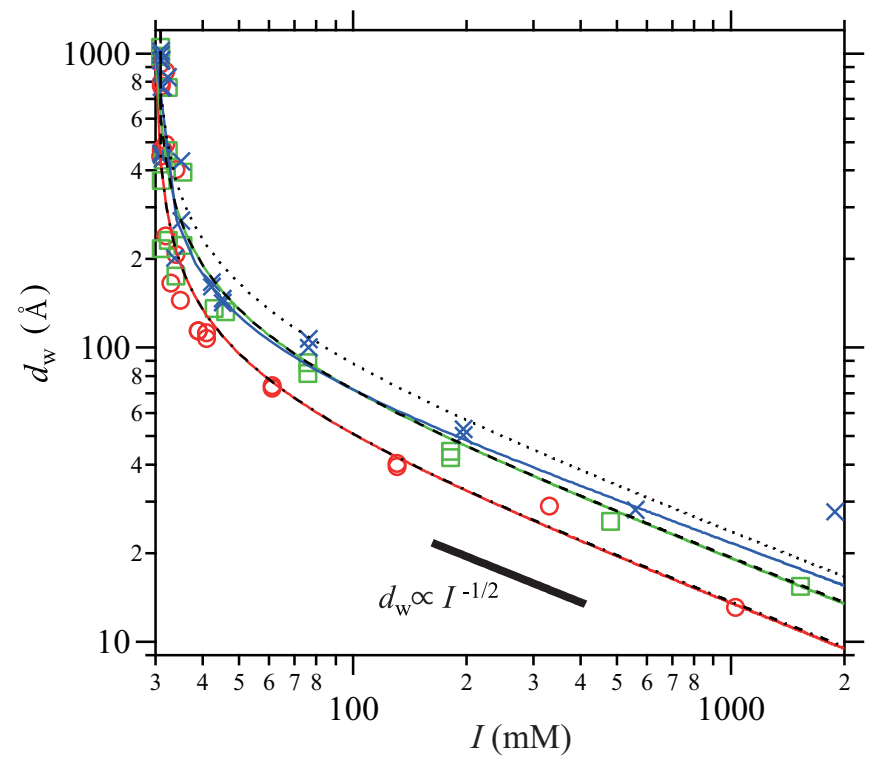

FIG. 2. Water layer thickness $d_{\mathrm{w}}$ as a function of the ionic strength $I$ in each saline solution $\left[\mathrm{NaCl}\right.$ (red circle), $\mathrm{Na}_{2} \mathrm{SO}_{4}$ (green square), $\mathrm{Na}_{3} \mathrm{PO}_{4}$ (blue cross)]. Colored solid lines represent the water layer thicknesses where $U\left(d_{\mathrm{w}}\right)$ [Eq. (10)] takes a minimum. Dash-dotted, dashed, and dotted lines are the plots of Eq. (11) for $\mathrm{NaCl}, \mathrm{Na}_{2} \mathrm{SO}_{4}$, and $\mathrm{Na}_{3} \mathrm{PO}_{4}$ solutions, respectively.

The Hamaker constant $H$, which determines the strength of the vdW interaction, is determined in terms of the dielectric constants of a lipid bilayer and an aqueous phase. Small changes in the lipid bilayer structure upon salt additions [Fig. 1(b)] imply that the dielectric constant of the lipid bilayer is also unchanged upon salt addition. In contrast, the dielectric constant of an aqueous phase changes upon salt addition. However, in the present concentration range (e.g., $\rho=100 \mathrm{mM}$ ), the change is very small and the salt-induced increase in $H$ from that in pure water is calculated to be within $1 \%$. Dependence of $H$ on the nature of the ions should therefore be negligible. In the present study, thus, we assume $H=4.7 \times 10^{-21} \mathrm{~J}$ [27] for all samples.

Since the classical DLVO theory $[3,8]$ predicts that $d_{\mathrm{w}}$ should be the same for the same $I$ and $H$ irrespective of the nature of the added ion, the dependence of $d_{\mathrm{w}}$ on the co-ion valence is not explained by it.

In addition to DLVO interactions (DL interaction and vdW interaction), two other interactions have been known to work between two flexible bilayers [22-25]: the Helfrich interaction due to fluctuation of the bilayer [28] and a short-range interaction sometimes called the hydration interaction [29]. The latter can be considered negligible in the present case, as it becomes obvious when $d_{\mathrm{w}}$ is less than $5 \AA$ [29]. The Helfrich interaction also has little effect in the present case, since it becomes dominant when $d_{\mathrm{w}}<20 \AA$ [3]. Further, the addition of ions has been reported to affect the bending rigidity of lipid bilayers only slightly [30], leading to little dependence on the ionic species involved in the Helfrich interaction. Since ion dependence of the van der Waals interaction was also negligible as stated above, it is concluded that the co-ion dependence of $d_{\mathrm{w}}$ results from the difference in the DL interactions.
Trefalt [16] suggested that co-ions are expelled from the gap between the colloid surfaces when the surface charge density $\sigma$ and co-ion valence $Z$ are large. $\sigma$ in the present case was calculated from the measured $\zeta$ potential using Graham's formula [3]. It was found that $\zeta$ does not depend on $I$ but on $\rho_{\mathrm{Na}}$ in the present systems, i.e., $\zeta$ is a function of $\rho_{\mathrm{Na}}$ irrespective of the co-ion species, and it fitted well with $\zeta / \mathrm{mV}=$ $18.0 \log _{10}\left(\rho_{\mathrm{Na}} / \mathrm{mM}\right)-145.3$. According to this formula, $\sigma$ in the present case was found to be -20 to $-60 \mathrm{mC} / \mathrm{m}^{2}$ for most of the $I$ range, which is comparable to the results obtained by Trefalt. We can expect that the co-ions are expelled from the DOPS lamellar phase accordingly. The added counterions are also expelled due to the charge neutrality. In this case, only the counterions originally stuck to the lipid remain between the bilayers, inducing the DL interaction: The DL interaction corresponds to the counter-ion-only case $[3,8]$. The osmotic pressure induced by the expelled ions also works, so that the pressure between two lipid bilayers becomes [16]

$$
P_{\mathrm{DL}}\left(d_{\mathrm{w}}\right)=\frac{2 \pi^{2} \varepsilon_{0} \varepsilon}{\beta^{2} e_{0}^{2}} \frac{1}{d_{\mathrm{w}}^{2}}-\frac{\rho_{\mathrm{anion}}^{\mathrm{add}}+\rho_{\mathrm{cation}}^{\mathrm{add}}}{\beta},
$$

where $\rho_{\text {anion }}^{\text {add }}$ and $\rho_{\text {cation }}^{\text {add }}$ are the concentrations of added anions and cations, respectively. The potential energy per unit area is given by the integral of $P_{\mathrm{DL}}\left(d_{\mathrm{w}}\right)$,

$$
\begin{aligned}
U_{\mathrm{DL}}\left(d_{\mathrm{w}}\right)= & \int_{d_{\mathrm{w}}}^{d_{\mathrm{w}}^{\max }} P_{\mathrm{DL}}(x) d x=\frac{2 \pi^{2} \varepsilon_{0} \varepsilon}{\beta^{2} e_{0}^{2}}\left(\frac{1}{d_{\mathrm{w}}}-\frac{1}{d_{\mathrm{w}}^{\max }}\right) \\
& +\frac{\rho_{\text {anion }}^{\text {add }}+\rho_{\text {cation }}^{\text {add }}}{\beta}\left(d_{\mathrm{w}}-d_{\mathrm{w}}^{\max }\right),
\end{aligned}
$$

where $d_{\mathrm{w}}^{\max }$ is the maximum limit of $d_{\mathrm{w}}$ determined by the volume fraction of the lipid in the solution $\left(d_{\mathrm{w}}^{\max } \approx 850 \AA\right.$ in the present case). For salts exhibiting simple and complete dissociation such as $\mathrm{NaCl}$ and $\mathrm{Na}_{2} \mathrm{SO}_{4}, \rho_{\text {anion }}^{\text {add }}+\rho_{\text {cation }}^{\text {add }}=(Z+$ 1) $\rho=\left(2 I-\rho_{0}\right) / Z$; thus,

$$
U_{\mathrm{DL}}\left(d_{\mathrm{w}}\right)=\frac{2 \pi^{2} \varepsilon_{0} \varepsilon}{\beta^{2} e_{0}^{2}}\left(\frac{1}{d_{\mathrm{w}}}-\frac{1}{d_{\mathrm{w}}^{\max }}\right)+\frac{2 I-\rho_{0}}{Z \beta}\left(d_{\mathrm{w}}-d_{\mathrm{w}}^{\max }\right) .
$$

The first term in Eq. (9) indicates a repulsive interaction independent of $Z$, while the second term is attractive and dependent on $Z$. For the same $I$ condition, the attractive interaction is stronger for a smaller $Z$, i.e., in the case of $\mathrm{NaCl}$ in the present study. We substituted $\rho_{\text {anion }}^{\text {add }}+\rho_{\text {cation }}^{\text {add }}=$ $4 \rho+\left(\sqrt{4 \rho K_{\mathrm{b}}+K_{\mathrm{b}}^{2}}-K_{\mathrm{b}}\right) / 2$ in Eq. (8) for the case with $\mathrm{Na}_{3} \mathrm{PO}_{4}$, taking into account the equilibrium between $\mathrm{PO}_{4}^{3-}$ and $\mathrm{HPO}_{4}^{2-}$.

By superposing the DL interaction and vdW interaction [Eq. (6)], the total potential energy per unit area is given by

$$
U\left(d_{\mathrm{w}}\right)=U_{\mathrm{DL}}\left(d_{\mathrm{w}}\right)+U_{\mathrm{vdW}}\left(d_{\mathrm{w}}\right) .
$$

The position of the potential minimum of $U\left(d_{\mathrm{w}}\right)$ (Fig. 3 for $I=300 \mathrm{mM}$ ) depends on the valence of the co-ion and it becomes larger with increasing valence. In Fig. 2, the calculated positions of the potential minima of $U\left(d_{\mathrm{w}}\right)$ are shown by colored solid lines. The calculations agree very 


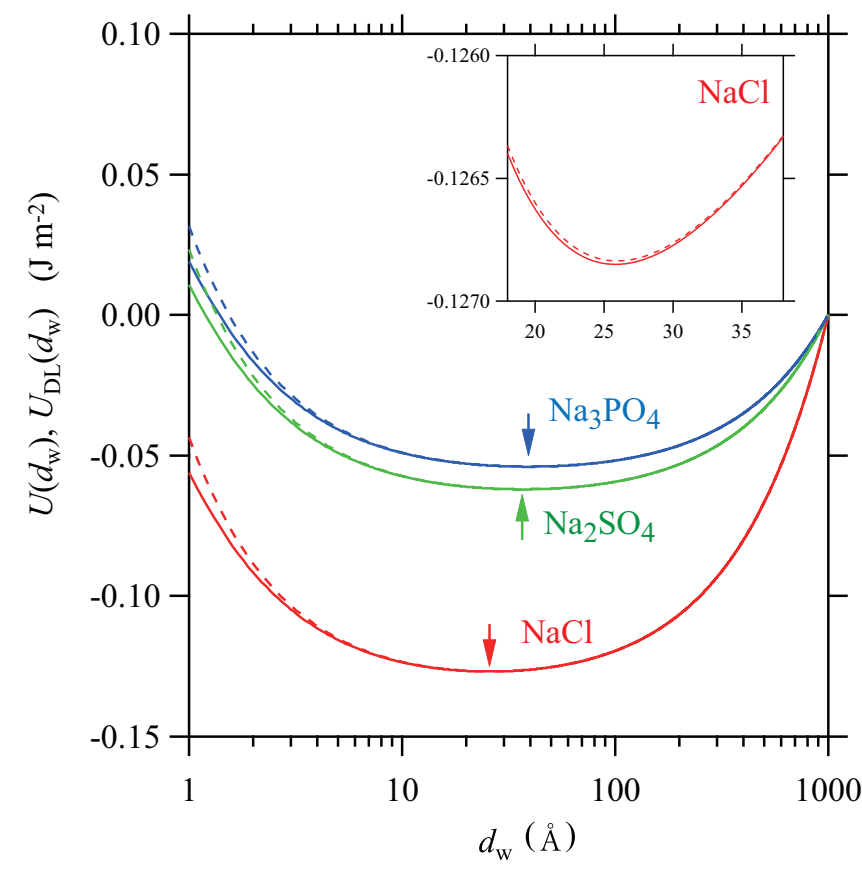

FIG. 3. Potential energies per unit area with $\left[U\left(d_{\mathrm{w}}\right)\right.$, solid lines $]$ and without $\left[U_{\mathrm{DL}}\left(d_{\mathrm{w}}\right)\right.$, dashed lines] van der Waals interaction when the ionic strength is $I=300 \mathrm{mM}$. $\mathrm{NaCl}$ (red), $\mathrm{Na}_{2} \mathrm{SO}_{4}$ (green), and $\mathrm{Na}_{3} \mathrm{PO}_{4}$ (blue). The arrows locate the minima. The inset shows the close-up around the minimum for co-ion valence 1 . The minima were located at 25.8 and $26.0 \AA$ for $U\left(d_{\mathrm{w}}\right)$ and $U_{\mathrm{DL}}\left(d_{\mathrm{w}}\right)$, respectively.

well with the experimental results for all samples, despite the absence of free parameters in this treatment, indicating that the co-ion dependence of $d_{\mathrm{w}}$ results from different osmotic pressures by the expulsion of added ions.

As shown in Fig. 3, $U_{\mathrm{DL}}\left(d_{\mathrm{w}}\right)$ is almost coincident with $U\left(d_{\mathrm{w}}\right)$, except for a smaller $d_{\mathrm{w}}$. The position of the potential minimum is determined almost completely by $U_{\mathrm{DL}}\left(d_{\mathrm{w}}\right)$, indicating that the vdW interaction is negligible and the main origin of the attractive force is the osmotic pressure due to the expelled ions. The vdW interaction becomes effective only when $d_{\mathrm{w}}$ is smaller than $10 \AA$.

Since the interaction potential between the charged lipid bilayers seems to be dominated only by $U_{\mathrm{DL}}\left(d_{\mathrm{w}}\right)$, the position of the potential minimum is easily calculated from $d U_{\mathrm{DL}}\left(d_{\mathrm{w}}\right) / d d_{\mathrm{w}}=0$, and the following formula is obtained:

$$
d_{\mathrm{w}}=\sqrt{\frac{2 \pi^{2} \varepsilon_{0} \varepsilon Z}{\beta e_{0}^{2}\left(2 I-\rho_{0}\right)}} .
$$

The black dash-dotted, dashed, and dotted lines in Fig. 2 are the plots corresponding to Eq. (11). These coincide well with the results where the vdW interaction was considered for the whole range of $d_{\mathrm{w}}$ considered in the present study. The mismatch was notable only in the case of $Z=3$ because Eq. (11) did not take into account the equilibrium between $\mathrm{PO}_{4}^{3-}$ and $\mathrm{HPO}_{4}^{2-}$. If the co-ion was only trivalent, the result would show better coincidence with the black dashed line. The $-1 / 2$ power low of the $I$ dependence of $d_{\mathrm{w}}$ can also be explained by Eq. (11), i.e., since $2 I-\rho_{0} \approx 2 I$ for a larger $I, d_{\mathrm{w}} \propto I^{-\frac{1}{2}}$.

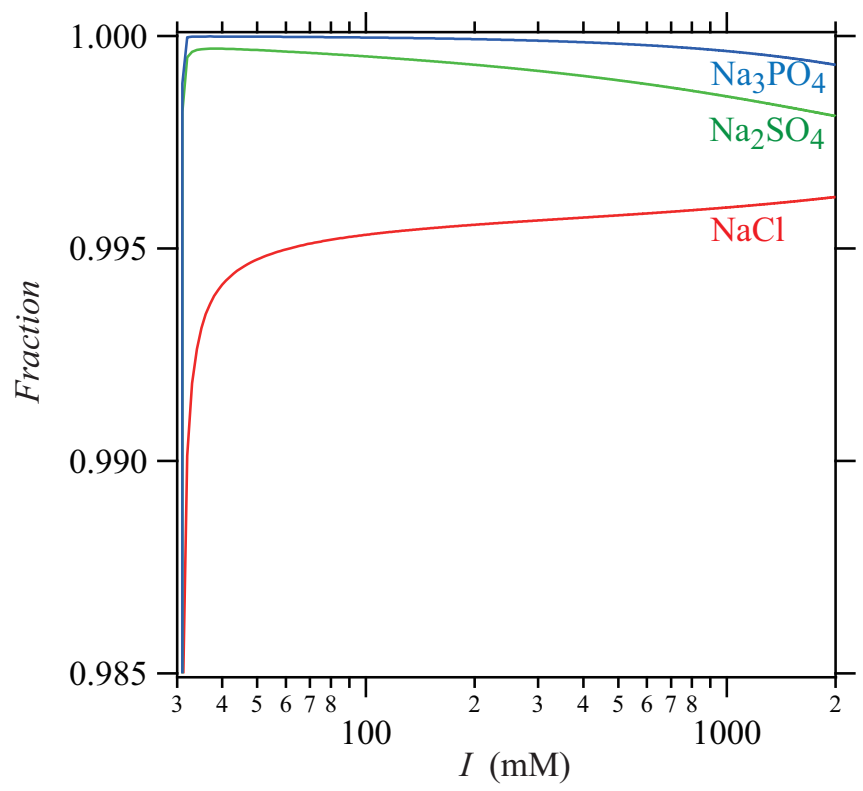

FIG. 4. Molecular fractions of co-ions in the bulk phase to the total co-ion added as functions of $I . \mathrm{NaCl}$ (red), $\mathrm{Na}_{2} \mathrm{SO}_{4}$ (green), and $\mathrm{Na}_{3} \mathrm{PO}_{4}$ (blue). Over $99 \%$ of added coions are expelled from the aqueous phase between bilayers.

The good agreement between the experiment and theory in the present study indicates that most of the added ions are expelled from the aqueous layer between lipid bilayers, even when the bilayer-bilayer distance is large. It is emphasized that the good agreement is seen even when $Z=1$ or $d_{\mathrm{w}}$ is rather large, in contrast to the reports of colloidal particles [15-19]. The phase diagram within the framework of the Poisson-Boltzmann treatment proposed by Andelman [8] suggests that strong expulsion of ions occurs when $\lambda_{\mathrm{D}} \gg d_{\mathrm{w}}$ and the Gouy-Chapman length $\lambda_{\mathrm{GC}}=2 \varepsilon_{0} \varepsilon / \beta e_{0}|\sigma| \ll d_{\mathrm{w}}$, i.e., $I$ is small and the surface charge density $\sigma$ is large ("Gouy-Chapman region," where the DL interaction coincides with counterions only). However, the calculated values for $\lambda_{\mathrm{GC}}$ and $\lambda_{\mathrm{D}}$ in the present case indicated that both are smaller than $d_{\mathrm{w}}$, i.e., $\lambda_{\mathrm{D}}$ is too short to match the Gouy-Chapman region.

On the other hand, the strong expulsion of the added salt is explained by the so-called Gibbs-Donnan effect [31] by considering the stacked bilayers as aggregated colloids in a virtual semipermeable membrane. Considering charge neutrality, the preservation of the number of ions, and the constancy of $\left[\mathrm{Na}^{+}\right]^{Z}\left[\mathrm{~A}^{Z-}\right]$ of the added salt between the lamellar phase and the bulk, while assuming the uniformity of the ion distribution in the lamellar phase, the fractions of co-ions in the bulk phase to the total added co-ions were calculated as shown in Fig. 4. (For the sake of ease, the equilibrium of $\mathrm{PO}_{4}^{3-}$ and $\mathrm{HPO}_{4}^{2-}$ was not considered here.) It is clear that most of the added co-ions exist in the bulk, implying added counterions are also expelled due to the charge neutrality. The multistacked nature of the lamellar phase seems significant for the Gibbs-Donnan effect to occur, in contrast to the system for the surface force measurements [10-12]. This is consistent with the fact that DL interactions seem different for multilayered bilayers and an isolated couple of bilayers, which follows the DLVO theory [10-12]. 
The present study demonstrates the importance of additional osmotic pressure owing to the expelled ions resulting in an attractive force. The origin of the osmotic pressure is entropy imbalance of water between the bulk phase and the aqueous phase between lipid bilayers. On the other hand, in the Poisson-Boltzmann treatment, the solvent is regarded as a continuum background and thermodynamics of the solvent is neglected. The present result indicates that the thermodynamic quantities of solvent need to be appropriately considered in order to understand the DL interaction.

This work was supported in part by a Grant-in-Aid for Scientific Research from Japan Society for the Promotion of Science (Grant No. 15K13546) to M.H. The x-ray scattering experiments were performed with the approval of the Photon Factory Program Advisory Committee (Proposals No. 2015G604 and No. 2015G571).
[1] B. V. Derjaguin and L. D. Landau, Acta Phys. Chim. URSS 14, 633 (1941).

[2] E. J. W. Verwey and J. Th. G. Overbeek, Theory of the Stability of Lyophobic Colloids (Dover, New York, 1999).

[3] J. N. Israelachvili, Intermolecular and Surface Forces, 3rd ed. (Academic Press, Amsterdam, 2010).

[4] G. Gouy, J. Phys. 9, 457 (1910).

[5] D. L. Chapman, Philos. Mag. 25, 475 (1913).

[6] P. Debye and E. Hückel, Phyzik Z. 24, 185 (1923).

[7] P. Debye and E. Hückel, Phyzik Z. 25, 97 (1924).

[8] D. Andelman, Handbook of Biological Physics (Elsevier, Amsterdam, 1995), Vol. 1, Chap. 12.

[9] X. Xing, Phys. Rev. E 83, 041410 (2011).

[10] J. Marra, J. Phys. Chem. 90, 2145 (1986).

[11] T. H. Anderson, S. H. Donaldson, H. Zeng, and J. N. Israelachvili, Langmuir 26, 14458 (2010).

[12] R. M. Pashley, P. M. McCuiggan, and B. W. Ninham, J. Phys. Chem. 90, 1637 (1986).

[13] G. Trefalt, I. Szilagyi, and M. Borkovec, J. Colloid Interface Sci. 406, 111 (2013).

[14] T. Cao, I. Szilagyi, T. Oncsik, M. Borkovec, and G. Trefalt, Langmuir 31, 6610 (2015).

[15] F. J. Montes Ruiz-Cabello, M. Moazzami-Gudarzi, M. Elzbieciak-Wodka, P. Maroni, C. Labbez, M. Borkovec, and G. Trefalt, Soft Matter 11, 1562 (2015).

[16] G. Trefalt, Phys. Rev. E 93, 032612 (2016).
[17] G. Trefalt, T. Palberg, and M. Borkovec, Curr. Opin. Colloid Interface Sci. 27, 9 (2017).

[18] G. Trefalt, I. Szilagyi, G. Téllez, and M. Borkovec, Langmuir 33, 1695 (2017).

[19] B. Uzelac, V. Valmacco, and G. Trefalt, Soft Matter 13, 5741 (2017).

[20] T. Saito, T. Iwaki, and K. Yoshikawa, Chem. Phys. Lett. 465, 40 (2008).

[21] T. Saito, T. Iwaki, and K. Yoshikawa, Biophys. J. 96, 1068 (2009).

[22] H. I. Petrache, N. Gouliaev, S. Tristram-Nagle, R. Zhang, R. M. Suter, and J. F. Nagle, Phys. Rev. E 57, 7014 (1998).

[23] M. Hishida, H. Seto, N. L. Yamada, and K. Yoshikawa, Chem. Phys. Lett. 455, 297 (2008).

[24] M. Hishida, Y. Yamamura, and K. Saito, Langmuir 30, 10583 (2014).

[25] M. Hishida, Y. Kaneko, Y. Yamamura, K. Saito, J. Solution Chem. 45, 1612 (2016).

[26] G. Pabst, M. Rappolt, H. Amenitsch, and P. Laggner, Phys. Rev. E 62, 4000 (2000).

[27] S. Tristram-Nagle, H. I. Petrache, and J. F. Nagle, Biophys. J. 75, 917 (1998).

[28] W. Helfrich, Z. Naturforsch. 33a, 305 (1978).

[29] J. Milhaud, Biochim. Biophys. Acta 1663, 19 (2004).

[30] H. I. Petrache, S. Tristram-Nagle, D. Harries, N. Kučerka, J. F. Nagle, and V. A. Parsegian, J. Lipid Res. 47, 302 (2006).

[31] F. G. Donnan, Chem. Rev. 1, 73 (1924). 